\title{
A $K$-SAMPLE RANK TEST BASED ON MODIFIED BAUMGARTNER STATISTIC AND ITS POWER COMPARISON
}

\author{
Hidetoshi Murakami*
}

\begin{abstract}
The purpose of this paper is to develop a nonparametric $k$-sample test based on a modified Baumgartner statistic. We define a new modified Baumgartner statistic $B^{*}$ and give some critical values. Then we compare the power of the $B^{*}$ statistic with the $t$-test, the Wilcoxon test, the Kolmogorov-Smirnov test, the Cramér-von Mises test, the Anderson-Darling test and the original Baumgartner statistic. The $B^{*}$ statistic is more suitable than the Baumgartner statistic for the location parameter when the sample sizes are not equal. Also, the $B^{*}$ statistic has almost the same power as the Wilcoxon test for location parameter. For scale parameter, the power of the $B^{*}$ statistic is more efficient than the Cramér-von Mises test and the Anderson-Darling test when the sizes are equal. The power of the $B^{*}$ statistic is higher than the Kolmogorov-Smirnov test for location and scale parameters. Then the $B^{*}$ statistic is generalized from two-sample to $k$-sample problems. The $B_{k}^{*}$ statistic denotes a $k$-sample statistic based on the $B^{*}$ statistic. We compare the power of the $B_{k}^{*}$ statistic with the Kruskal-Wallis test, the $k$-sample Kolmogorov-Smirnov test, the $k$-sample Cramér-von Mises test, the $k$-sample Anderson-Darling test and the $k$-sample Baumgartner statistic. Finally, we investigate the behavior of power about the $B_{k}^{*}$ statistics by simulation studies. As a result, we obtain that the $B_{k}^{*}$ statistic is more suitable than the other statistics.
\end{abstract}

\section{Introduction}

Let $\boldsymbol{X}=\left(X_{1}, \ldots, X_{n}\right)$ and $\boldsymbol{Y}=\left(Y_{1}, \ldots, Y_{m}\right)$ be two random samples of size $n$ and $m$ independent observations from different populations with continuous distribution $F(x)$ and $G(y)$, respectively. The well known test of Wilcoxon (Hollander and Wolfe, 1999) is a test for location as $F(x)=G(y-\theta)$. Baumgartner, Weiß and Schindler (1998) presented a new nonparametric two-sample test which has almost same power as the Wilcoxon test for the location parameter. They assert that the Baumgartner statistic can be applied for the scale parameter as $F(x)=G\left(\frac{y}{\sigma}\right)$, where $\sigma \neq 0$. Let $R_{1}<\cdots<R_{n}$ and $H_{1}<\cdots<H_{m}$ denote the combined-samples ranks of the $X$-values and $Y$-values in increasing order of magnitude, respectively. The test statistic $B$ proposed by Baumgartner et al. (1998) is

$$
B=\frac{1}{2}\left(B_{X}+B_{Y}\right)
$$

where

$$
B_{\mathrm{X}}=\frac{1}{n} \sum_{i=1}^{n} \frac{\left(R_{i}-\frac{n+m}{n} i\right)^{2}}{\frac{i}{n+1}\left(1-\frac{i}{n+1}\right) \frac{m(n+m)}{n}}
$$

\footnotetext{
*Department of Mathematics, Chuo University, 1-13-27 Kasuga, Bunkyo-ku, Tokyo 112-8551, Japan E-mail: murakami@gug.math.chuo-u.ac.jp

Key words: $K$-sample power comparison; Nonparametric
} 
and

$$
B_{\mathrm{Y}}=\frac{1}{m} \sum_{j=1}^{m} \frac{\left(H_{j}-\frac{m+n}{m} j\right)^{2}}{\frac{j}{m+1}\left(1-\frac{j}{m+1}\right) \frac{n(m+n)}{m}} .
$$

Recently, Neuhäuser (2003) researched ties of the Baumgartner statistic. Also, Neuhäuser (2000) suggested an exact two-sample test based on the Baumgartner statistic. In this paper, first, we propose a new nonparametric test by modifying the Baumgartner statistic. Second, we extend the new statistic to the $k$-sample problem. Finally, to compare the new method with previous methods, we will investigate its power by simulation study.

In Section 2, we consider the modified Baumgartner statistic $B^{*}$ and compare the power of the $B^{*}$ statistic with the $t$-test, the Wilcoxon test $W$, the Kolmogorov-Smirnov test $K S$ (Hollander and Wolfe, 1999), the Cramér-von Mises test $C V$ (Anderson, 1962), the Anderson-Darling test $A D$ (Pettitt, 1976) and the $B$ statistic. Some critical values of the $B^{*}$ statistic are listed in Table 1 . We know that the $B^{*}$ statistic is more efficient than the $B$ statistic when $n \neq m$. In Section 3 , since the $k$-sample problems are important in statistical practice, we propose the statistic $B_{k}^{*}$ for the $k$-sample test and investigate the behavior of the $B_{k}^{*}$ statistic. Then, we compare the power of the $B_{k}^{*}$ statistic with the Kruskal-Wallis test $W_{k}$ (Hájek, Šidák and Sen, 1999), the $k$-sample Kolmogorov-Smirnov test $K S_{k}$ (Hájek et al., 1999 ), the $k$-sample Cramér-von Mises test $C V_{k}$ (Kiefer, 1959), the $k$-sample AndersonDarling test $A D_{k}$ (Scholz and Stephens, 1987) and the $k$-sample Baumgartner statistic $B_{k}$. For comparison of the statistics, we perform simulation studies using the normal, double exponential, logistic and exponential distributions as different populations. We perform the simulation studies for $k=3$. For all simulation studies, we have 1,000,000 times repetition.

\section{Modified Baumgartner statistic}

In this section, we propose the modified Baumgartner statistic $B^{*}$, and compare the power with the $t$-test, the $W$ statistic, the $K S$ statistic, the $C V$ statistic, the $A D$ statistic and the $B$ statistic by simulation studies. It is well known that the expected value and variance of $R_{i}$ are

$$
\mathrm{E}\left[R_{i}\right]=\frac{n+m+1}{n+1} i
$$

and

$$
\operatorname{Var}\left[R_{i}\right]=\frac{i}{n+1}\left(1-\frac{i}{n+1}\right) \frac{m(n+m+1)}{n+2},
$$

respectively (Arnold, Balakrishnan and Nagaraja, 1992). Similarly, the expected value and variance of $H_{j}$ are

$$
\mathrm{E}\left[H_{j}\right]=\frac{m+n+1}{m+1} j
$$

and

$$
\operatorname{Var}\left[H_{j}\right]=\frac{j}{m+1}\left(1-\frac{j}{m+1}\right) \frac{n(m+n+1)}{m+2} .
$$

Here, the modified Baumgartner statistic $B^{*}$ is defined by

$$
B^{*}=\frac{1}{2}\left(B_{X}^{*}+B_{Y}^{*}\right),
$$


where

$$
B_{\mathrm{X}}^{*}=\frac{1}{n} \sum_{i=1}^{n} \frac{\left(R_{i}-\mathrm{E}\left[R_{i}\right]\right)^{2}}{\operatorname{Var}\left[R_{i}\right]}
$$

and

$$
B_{\mathrm{Y}}^{*}=\frac{1}{m} \sum_{j=1}^{m} \frac{\left(H_{j}-\mathrm{E}\left[H_{j}\right]\right)^{2}}{\operatorname{Var}\left[H_{j}\right]}
$$

Now, we compare the power of the $B^{*}$ statistic with some other statistics before we elaborate. Suppose that $F(x)$ and $G(y)$ are distributed as $N\left(\mu_{1}, \sigma_{1}^{2}\right)$ and $N\left(\mu_{2}, \sigma_{2}^{2}\right), D E\left(\mu_{1}, \sigma_{1}\right)$ and $D E\left(\mu_{2}, \sigma_{2}\right), L\left(\mu_{1}, \sigma_{1}\right)$ and $L\left(\mu_{2}, \sigma_{2}\right)$, and $\eta\left(\lambda_{1}\right)$ and $\eta\left(\lambda_{2}\right)$, respectively. $N\left(\mu, \sigma^{2}\right)$ denotes the normal distribution with mean $\mu$ and variance $\sigma^{2} . D E(\mu, \sigma)$ and $L(\mu, \sigma)$ denote the double exponential and logistic distributions with mean $\mu$ and variance $\sigma$, respectively. Also, $\eta(\lambda)$ denotes the exponential distribution with the mean parameter $\lambda$. Neuhäuser (2000) calculated the critical values of the $B$ statistic $(\operatorname{Pr}(B \geq b))$ for equal sample sizes only. The critical values of $B$ statistic and $B^{*}$ statistic are listed in Table 1 including different sample sizes for simulation studies. Baumgartner et al. (1998) derived the asymptotic distribution, and its critical values were $\operatorname{Pr}(B \geq 3.880)=0.010$ and $\operatorname{Pr}(B \geq 2.493)=0.050$. The asymptotic distribution of the $B^{*}$ statistic is same as the $B$ statistic. From the Table 1 , we can easily see that $b^{*}$ converges to the point of the asymptotic distribution.

Table 1-(a): Exact critical value

\begin{tabular}{cc|cc|cc|cc|cc}
\hline$n$ & $m$ & $\operatorname{Pr}(B \geq b)$ & $b$ & $\operatorname{Pr}(B \geq b)$ & $b$ & $\operatorname{Pr}\left(B^{*} \geq b^{*}\right)$ & $b^{*}$ & $\operatorname{Pr}\left(B^{*} \geq b^{*}\right)$ & $b^{*}$ \\
\hline 5 & 5 & 0.0079 & 4.894 & 0.0476 & 2.533 & 0.0079 & 5.536 & 0.0476 & 2.870 \\
10 & 5 & 0.0100 & 4.033 & 0.0496 & 2.552 & 0.0100 & 4.326 & 0.0500 & 2.703 \\
10 & 10 & 0.0100 & 4.120 & 0.0500 & 2.583 & 0.0100 & 4.359 & 0.0500 & 2.700 \\
\hline
\end{tabular}

Table 1-(b): Critical value by simulation

\begin{tabular}{cc|cc|cc|cc|cc}
\hline$n$ & $m$ & $\operatorname{Pr}(B \geq b)$ & $b$ & $\operatorname{Pr}(B \geq b)$ & $b$ & $\operatorname{Pr}\left(B^{*} \geq b^{*}\right)$ & $b^{*}$ & $\operatorname{Pr}\left(B^{*} \geq b^{*}\right)$ & $b^{*}$ \\
\hline 10 & 10 & 0.0100 & 4.119 & 0.0500 & 2.583 & 0.0100 & 4.359 & 0.0500 & 2.700 \\
20 & 20 & 0.0100 & 4.129 & 0.0500 & 2.596 & 0.0100 & 4.219 & 0.0500 & 2.633 \\
30 & 30 & 0.0100 & 4.087 & 0.0500 & 2.584 & 0.0100 & 4.144 & 0.0500 & 2.604 \\
40 & 40 & 0.0100 & 4.063 & 0.0500 & 2.576 & 0.0100 & 4.097 & 0.0500 & 2.578 \\
50 & 50 & 0.0100 & 4.021 & 0.0500 & 2.565 & 0.0100 & 4.081 & 0.0500 & 2.572 \\
100 & 100 & 0.0100 & 3.962 & 0.0500 & 2.535 & 0.0100 & 3.991 & 0.0500 & 2.539 \\
200 & 200 & 0.0100 & 3.929 & 0.0500 & 2.522 & 0.0100 & 3.937 & 0.0500 & 2.523 \\
300 & 300 & 0.0100 & 3.910 & 0.0500 & 2.512 & 0.0100 & 3.905 & 0.0500 & 2.504 \\
\hline
\end{tabular}

The values in Tables 2 to 5 are results for the case of $\mu_{1} \neq \mu_{2}$ and $\sigma_{1}=\sigma_{2}$ or $\mu_{1}=\mu_{2}$ and $\sigma_{1} \neq \sigma_{2}$ when $n=m$ and $n \neq m$, respectively, and the significant level is $5 \%$. 
Table 2-(a): The case of $n=m=10$ for $N(0,1)$ and $N\left(\mu_{2}, 1\right)$

\begin{tabular}{cccccccc}
\hline & & & \multicolumn{5}{c}{$\mu_{2}$} \\
\cline { 2 - 7 } & 0.0 & 0.5 & 1.0 & 1.5 & 2.0 & 2.5 & 3.0 \\
\hline$t$ & $\mathbf{0 . 0 5}$ & 0.19 & 0.56 & 0.89 & 0.99 & 1.00 & 1.00 \\
$W$ & $\mathbf{O . 0 5}$ & 0.18 & 0.54 & 0.86 & 0.98 & 1.00 & 1.00 \\
$K S$ & $\mathbf{O . 0 5}$ & 0.16 & 0.47 & 0.80 & 0.96 & 1.00 & 1.00 \\
$C M$ & $\mathbf{O . 0 5}$ & 0.17 & 0.52 & 0.85 & 0.98 & 1.00 & 1.00 \\
$A D$ & $\mathbf{O . 0 5}$ & 0.17 & 0.52 & 0.86 & 0.98 & 1.00 & 1.00 \\
$B$ & $\mathbf{O . 0 5}$ & 0.17 & 0.52 & 0.85 & 0.98 & 1.00 & 1.00 \\
$B^{*}$ & $\mathbf{O . 0 5}$ & 0.17 & 0.52 & 0.85 & 0.98 & 1.00 & 1.00 \\
\hline
\end{tabular}

Table 2-(b): The case of $n=m=10$ for $N(0,1)$ and $N\left(0, \sigma_{2}^{2}\right)$

\begin{tabular}{cccccccc}
\hline & & & \multicolumn{7}{c}{$\sigma_{2}$} \\
\cline { 2 - 8 } & 1.0 & 5.0 & 10.0 & 15.0 & 20.0 & 25.0 & 30.0 \\
\hline$W$ & $\mathbf{O . 0 5}$ & 0.08 & 0.09 & 0.10 & 0.10 & 0.10 & 0.10 \\
$K S$ & $\mathbf{O . 0 5}$ & 0.32 & 0.49 & 0.57 & 0.61 & 0.64 & 0.66 \\
$C M$ & $\mathbf{O . 0 5}$ & 0.23 & 0.39 & 0.48 & 0.53 & 0.57 & 0.60 \\
$A D$ & $\mathbf{O . 0 5}$ & 0.30 & 0.54 & 0.66 & 0.74 & 0.78 & 0.82 \\
$B$ & $\mathbf{O . 0 5}$ & 0.34 & 0.58 & 0.70 & 0.77 & 0.81 & 0.84 \\
$B^{*}$ & $\mathbf{O . 0 5}$ & 0.33 & 0.58 & 0.70 & 0.77 & 0.81 & 0.84 \\
\hline
\end{tabular}

Table 2-(c): The case of $n=10, m=5$ for $N(0,1)$ and $N\left(\mu_{2}, 1\right)$

\begin{tabular}{cccccccc}
\hline & & \multicolumn{7}{c}{$\mu_{2}$} \\
\cline { 2 - 7 } & 0.0 & 0.5 & 1.0 & 1.5 & 2.0 & 2.5 & 3.0 \\
\hline$t$ & $\mathbf{0 . 0 5}$ & 0.14 & 0.39 & 0.72 & 0.92 & 0.99 & 1.00 \\
$W$ & $\mathbf{O . 0 5}$ & 0.13 & 0.38 & 0.70 & 0.90 & 0.98 & 0.99 \\
$K S$ & $\mathbf{O . 0 6}$ & 0.13 & 0.36 & 0.64 & 0.86 & 0.96 & 0.99 \\
$C M$ & $\boldsymbol{O . 0 5}$ & 0.13 & 0.36 & 0.67 & 0.89 & 0.98 & 1.00 \\
$A D$ & $\mathbf{O . 0 5}$ & 0.13 & 0.36 & 0.67 & 0.89 & 0.98 & 1.00 \\
$B$ & $\mathbf{O . 0 5}$ & 0.10 & 0.31 & 0.62 & 0.86 & 0.97 & 1.00 \\
$B^{*}$ & $\boldsymbol{O . 0 5}$ & 0.13 & 0.36 & 0.67 & 0.89 & 0.98 & 1.00 \\
\hline
\end{tabular}

Table 2-(d): The case of $n=10, m=5$ for $N(0,1)$ and $N\left(0, \sigma_{2}^{2}\right)$

\begin{tabular}{cccccccc}
\hline & & & \multicolumn{7}{c}{$\sigma_{2}$} \\
\cline { 2 - 8 } & 1.0 & 5.0 & 10.0 & 15.0 & 20.0 & 25.0 & 30.0 \\
\hline$W$ & $\mathbf{O . 0 5}$ & 0.10 & 0.08 & 0.08 & 0.07 & 0.07 & 0.07 \\
$K S$ & $\mathbf{O . 0 6}$ & 0.22 & 0.29 & 0.31 & 0.33 & 0.34 & 0.34 \\
$C M$ & $\mathbf{O . 0 5}$ & 0.21 & 0.28 & 0.31 & 0.33 & 0.34 & 0.34 \\
$A D$ & $\boldsymbol{O . 0 5}$ & 0.23 & 0.30 & 0.32 & 0.34 & 0.34 & 0.35 \\
$B$ & $\mathbf{O . 0 5}$ & 0.22 & 0.29 & 0.31 & 0.33 & 0.34 & 0.34 \\
$B^{*}$ & $\mathbf{O . 0 5}$ & 0.22 & 0.29 & 0.31 & 0.33 & 0.34 & 0.34 \\
\hline
\end{tabular}


A $K$-sample Baumgartner Statistic

Table 3-(a): The case of $n=m=10$ for $D E(0,1)$ and $D E\left(\mu_{2}, 1\right)$

\begin{tabular}{cccccccc}
\hline & & & & $\mu_{2}$ & & & \\
\cline { 2 - 7 } & 0.0 & 0.5 & 1.0 & 1.5 & 2.0 & 2.5 & 3.0 \\
\hline$W$ & $\mathbf{0 . 0 5}$ & 0.15 & 0.42 & 0.71 & 0.88 & 0.96 & 0.99 \\
$K S$ & $\boldsymbol{O . 0 5}$ & 0.15 & 0.43 & 0.71 & 0.89 & 0.96 & 0.99 \\
$C M$ & $\boldsymbol{O . 0 5}$ & 0.15 & 0.42 & 0.72 & 0.89 & 0.97 & 0.99 \\
$A D$ & $\boldsymbol{O . 0 5}$ & 0.15 & 0.42 & 0.71 & 0.89 & 0.97 & 0.99 \\
$B$ & $\boldsymbol{O . 0 5}$ & 0.15 & 0.42 & 0.71 & 0.89 & 0.97 & 0.99 \\
$B^{*}$ & $\boldsymbol{O . 0 5}$ & 0.15 & 0.42 & 0.71 & 0.89 & 0.97 & 0.99 \\
\hline
\end{tabular}

Table 3-(b): The case of $n=m=10$ for $D E(0,1)$ and $D E\left(0, \sigma_{2}\right)$

\begin{tabular}{cccccccc}
\hline & & & \multicolumn{7}{c}{$\sigma_{2}$} \\
\cline { 2 - 8 } & 1.0 & 3.0 & 5.0 & 7.0 & 9.0 & 11.0 & 13.0 \\
\hline$W$ & $\mathbf{O . 0 5}$ & 0.06 & 0.07 & 0.08 & 0.09 & 0.09 & 0.10 \\
$K S$ & $\boldsymbol{O . 0 5}$ & 0.13 & 0.23 & 0.31 & 0.36 & 0.41 & 0.45 \\
$C M$ & $\boldsymbol{O . 0 5}$ & 0.10 & 0.17 & 0.22 & 0.27 & 0.31 & 0.35 \\
$A D$ & $\boldsymbol{O . 0 5}$ & 0.11 & 0.20 & 0.29 & 0.37 & 0.43 & 0.49 \\
$B$ & $\mathbf{O . 0 5}$ & 0.12 & 0.23 & 0.32 & 0.40 & 0.47 & 0.52 \\
$B^{*}$ & $\mathbf{O . 0 5}$ & 0.12 & 0.22 & 0.32 & 0.40 & 0.46 & 0.52 \\
\hline
\end{tabular}

Table 3-(c): The case of $n=10, m=5$ for $D E(0,1)$ and $D E\left(\mu_{2}, 1\right)$

\begin{tabular}{cccccccc}
\hline & & & \multicolumn{7}{c}{$\mu_{2}$} \\
\cline { 2 - 8 } & 0.0 & 0.5 & 1.0 & 1.5 & 2.0 & 2.5 & 3.0 \\
\hline$W$ & $\boldsymbol{0 . 0 5}$ & 0.12 & 0.30 & 0.52 & 0.71 & 0.84 & 0.92 \\
$K S$ & $\mathbf{0 . 0 6}$ & 0.13 & 0.32 & 0.55 & 0.74 & 0.87 & 0.93 \\
$C M$ & $\boldsymbol{O . 0 5}$ & 0.11 & 0.29 & 0.52 & 0.72 & 0.86 & 0.93 \\
$A D$ & $\boldsymbol{O . 0 5}$ & 0.11 & 0.29 & 0.52 & 0.72 & 0.86 & 0.93 \\
$B$ & $\boldsymbol{O . 0 5}$ & 0.09 & 0.25 & 0.47 & 0.68 & 0.83 & 0.92 \\
$B^{*}$ & $\boldsymbol{O . 0 5}$ & 0.12 & 0.30 & 0.52 & 0.72 & 0.86 & 0.93 \\
\hline
\end{tabular}

Table 3-(d): The case of $n=10, m=5$ for $D E(0,1)$ and $D E\left(0, \sigma_{2}\right)$

\begin{tabular}{cccccccc}
\hline & & & & $\sigma_{2}$ & & & \\
\cline { 2 - 8 } & 1.0 & 3.0 & 5.0 & 7.0 & 9.0 & 11.0 & 13.0 \\
\hline$W$ & $\mathbf{0 . 0 5}$ & 0.09 & 0.10 & 0.10 & 0.10 & 0.09 & 0.09 \\
$K S$ & $\mathbf{0 . 0 6}$ & 0.13 & 0.18 & 0.22 & 0.24 & 0.26 & 0.28 \\
$C M$ & $\mathbf{0 . 0 5}$ & 0.12 & 0.17 & 0.21 & 0.24 & 0.26 & 0.27 \\
$A D$ & $\mathbf{O . 0 5}$ & 0.13 & 0.19 & 0.23 & 0.26 & 0.28 & 0.29 \\
$B$ & $\mathbf{0 . 0 5}$ & 0.12 & 0.18 & 0.21 & 0.24 & 0.26 & 0.27 \\
$B^{*}$ & $\mathbf{0 . 0 5}$ & 0.12 & 0.18 & 0.21 & 0.24 & 0.26 & 0.27 \\
\hline
\end{tabular}


MURAKAMI

Table 4-(a): The case of $n=m=10$ for $L(0,1)$ and $L\left(\mu_{2}, 1\right)$

\begin{tabular}{cccccccc}
\hline & & & \multicolumn{7}{c}{$\mu_{2}$} \\
\cline { 2 - 8 } & 0.0 & 0.5 & 1.0 & 1.5 & 2.0 & 2.5 & 3.0 \\
\hline$W$ & $\mathbf{0 . 0 5}$ & 0.09 & 0.23 & 0.44 & 0.67 & 0.84 & 0.93 \\
$K S$ & $\mathbf{O . 0 5}$ & 0.09 & 0.21 & 0.41 & 0.62 & 0.80 & 0.91 \\
$C M$ & $\mathbf{O . 0 5}$ & 0.09 & 0.22 & 0.43 & 0.66 & 0.83 & 0.93 \\
$A D$ & $\mathbf{O . 0 5}$ & 0.09 & 0.22 & 0.43 & 0.66 & 0.83 & 0.93 \\
$B$ & $\mathbf{0 . 0 5}$ & 0.09 & 0.22 & 0.43 & 0.65 & 0.83 & 0.93 \\
$B^{*}$ & $\mathbf{0 . 0 5}$ & 0.09 & 0.22 & 0.43 & 0.66 & 0.83 & 0.93 \\
\hline
\end{tabular}

Table 4-(b): The case of $n=m=10$ for $L(0,1)$ and $L\left(0, \sigma_{2}\right)$

\begin{tabular}{cccccccc}
\hline & & \multicolumn{7}{c}{$\sigma_{2}$} \\
\cline { 2 - 8 } & 1.0 & 3.0 & 5.0 & 7.0 & 9.0 & 11.0 & 13.0 \\
\hline$W$ & $\mathbf{0 . 0 5}$ & 0.06 & 0.08 & 0.08 & 0.09 & 0.09 & 0.09 \\
$K S$ & $\mathbf{0 . 0 5}$ & 0.16 & 0.29 & 0.38 & 0.44 & 0.49 & 0.52 \\
$C M$ & $\mathbf{0 . 0 5}$ & 0.12 & 0.21 & 0.28 & 0.34 & 0.39 & 0.42 \\
$A D$ & $\mathbf{0 . 0 5}$ & 0.14 & 0.27 & 0.39 & 0.47 & 0.53 & 0.59 \\
$B$ & $\mathbf{0 . 0 5}$ & 0.16 & 0.31 & 0.42 & 0.51 & 0.58 & 0.63 \\
$B^{*}$ & $\mathbf{0 . 0 5}$ & 0.16 & 0.30 & 0.42 & 0.51 & 0.57 & 0.63 \\
\hline
\end{tabular}

Table 4-(c): The case of $n=10, m=5$ for $L(0,1)$ and $L\left(\mu_{2}, 1\right)$

\begin{tabular}{cccccccc}
\hline & & & \multicolumn{7}{c}{$\mu_{2}$} \\
\cline { 2 - 8 } & 0.0 & 0.5 & 1.0 & 1.5 & 2.0 & 2.5 & 3.0 \\
\hline$W$ & $\boldsymbol{O . 0 5}$ & 0.08 & 0.17 & 0.31 & 0.49 & 0.66 & 0.79 \\
$K S$ & $\boldsymbol{O . 0 6}$ & 0.08 & 0.17 & 0.30 & 0.47 & 0.64 & 0.78 \\
$C M$ & $\boldsymbol{O . 0 5}$ & 0.08 & 0.16 & 0.30 & 0.47 & 0.64 & 0.79 \\
$A D$ & $\boldsymbol{O . 0 5}$ & 0.08 & 0.16 & 0.30 & 0.47 & 0.65 & 0.79 \\
$B$ & $\boldsymbol{O . 0 5}$ & 0.06 & 0.13 & 0.26 & 0.41 & 0.59 & 0.74 \\
$B^{*}$ & $\boldsymbol{O . 0 5}$ & 0.08 & 0.16 & 0.30 & 0.47 & 0.65 & 0.79 \\
\hline
\end{tabular}

Table 4 -(d): The case of $n=10, m=5$ for $L(0,1)$ and $L\left(0, \sigma_{2}\right)$

\begin{tabular}{cccccccc}
\hline & & & \multicolumn{7}{c}{$\sigma_{2}$} \\
\cline { 2 - 8 } & 1.0 & 3.0 & 5.0 & 7.0 & 9.0 & 11.0 & 13.0 \\
\hline$W$ & $\boldsymbol{O . 0 5}$ & 0.09 & 0.10 & 0.09 & 0.09 & 0.09 & 0.08 \\
$K S$ & $\boldsymbol{O . 0 6}$ & 0.15 & 0.21 & 0.25 & 0.27 & 0.29 & 0.30 \\
$C M$ & $\mathbf{O . 0 5}$ & 0.14 & 0.20 & 0.24 & 0.27 & 0.29 & 0.30 \\
$A D$ & $\boldsymbol{O . 0 5}$ & 0.15 & 0.22 & 0.26 & 0.29 & 0.30 & 0.31 \\
$B$ & $\boldsymbol{O . 0 5}$ & 0.14 & 0.20 & 0.24 & 0.27 & 0.29 & 0.30 \\
$B^{*}$ & $\boldsymbol{O . 0 5}$ & 0.14 & 0.20 & 0.24 & 0.27 & 0.29 & 0.30 \\
\hline
\end{tabular}


Table 5-(a): The case of $n=m=10$ for $\eta(1)$ and $\eta\left(\lambda_{2}\right)$

\begin{tabular}{cccccccc}
\hline & & & \multicolumn{7}{c}{$\lambda_{2}$} \\
\cline { 2 - 8 } & 1.0 & 2.0 & 3.0 & 4.0 & 5.0 & 6.0 & 7.0 \\
\hline$W$ & $\mathbf{0 . 0 5}$ & 0.24 & 0.51 & 0.69 & 0.80 & 0.87 & 0.91 \\
$K S$ & $\mathbf{0 . 0 5}$ & 0.22 & 0.46 & 0.64 & 0.77 & 0.84 & 0.90 \\
$C M$ & $\mathbf{0 . 0 5}$ & 0.23 & 0.49 & 0.68 & 0.80 & 0.87 & 0.91 \\
$A D$ & $\mathbf{0 . 0 5}$ & 0.24 & 0.50 & 0.69 & 0.81 & 0.88 & 0.92 \\
$B$ & $\mathbf{0 . 0 5}$ & 0.24 & 0.51 & 0.70 & 0.81 & 0.89 & 0.92 \\
$B^{*}$ & $\mathbf{0 . 0 5}$ & 0.24 & 0.50 & 0.70 & 0.81 & 0.88 & 0.92 \\
\hline
\end{tabular}

Table 5-(b): The case of $n=10, m=5$ for $\eta(1)$ and $\eta\left(\lambda_{2}\right)$

\begin{tabular}{cccccccc}
\hline & & \multicolumn{7}{c}{$\lambda_{2}$} \\
\cline { 2 - 8 } & 1.0 & 2.0 & 3.0 & 4.0 & 5.0 & 6.0 & 7.0 \\
\hline$W$ & $\mathbf{0 . 0 5}$ & 0.19 & 0.37 & 0.51 & 0.61 & 0.69 & 0.74 \\
$K S$ & $\mathbf{O . 0 6}$ & 0.18 & 0.34 & 0.47 & 0.58 & 0.66 & 0.72 \\
$C M$ & $\mathbf{O . 0 5}$ & 0.18 & 0.35 & 0.50 & 0.60 & 0.69 & 0.75 \\
$A D$ & $\boldsymbol{O . 0 5}$ & 0.18 & 0.37 & 0.52 & 0.63 & 0.70 & 0.76 \\
$B$ & $\mathbf{O . 0 5}$ & 0.15 & 0.32 & 0.46 & 0.57 & 0.66 & 0.72 \\
$B^{*}$ & $\mathbf{O . 0 5}$ & 0.18 & 0.36 & 0.51 & 0.61 & 0.69 & 0.75 \\
\hline
\end{tabular}

From the Tables, we can see that the power of the $B$ and $B^{*}$ statistics are almost equal for the location test when $n=m$. However, the power of the $B^{*}$ statistic is greater than that of the $B$ statistic when $n \neq m$ for the location test. For the case of $\mu_{1}=\mu_{2}, \sigma_{1} \neq \sigma_{2}$, both the $B$ and $B^{*}$ statistics have almost equal power when $n=m$ or $n \neq m$. For a location parameter, the $B^{*}$ statistic is almost the same as the Wilcoxon test, and the $B^{*}$ statistic is more efficient than the Kolmogorov-Smirnov test for location and scale parameters. Also, the $B^{*}$ statistic is more suitable for a scale parameter than the Cramér-von Mises test and the Anderson-Darling test when $n=m$.

\section{Generalization of the modified Baumgartner statistic $B^{*}$}

In Section 2, we have obtained the result that the $B^{*}$ statistic is superior to the $B$ statistic. Now, we generalize the modified Baumgartner statistic $B^{*}$ from the two-sample to $k$-sample test. Recently, Neuhäuser (2001) considered a trend test based on the Baumgartner statistic for pairwise comparison.

Let $\left\{X_{i j} \mid i=1, \ldots, k, j=1, \ldots, n_{i}\right\}$ be $k$ independent samples of size $n_{1}, \ldots, n_{k}$ of independent observations. The observation $X_{i j}$ is assumed to be obtained from a continuous distribution function $F_{i}(x)$. Let $R_{i j}$ be the increasing-order rank of $X_{i j}$ in the combined $N=n_{1}+\cdots+n_{k}$ samples. One of the problems is to test the hypothesis $H_{0}: F_{1}=F_{2}=$ $\cdots=F_{k}$ against $H_{1}$ : not $H_{0}$. Let $B_{k}^{*}$ be the $k$-sample modified Baumgartner statistic defined by

$$
B_{k}^{*}=\frac{1}{k} \sum_{i=1}^{k}\left\{\frac{1}{n_{i}} \sum_{j=1}^{n_{i}} \frac{\left(R_{i j}-\mathrm{E}\left[R_{i j}\right]\right)^{2}}{\operatorname{Var}\left[R_{i j}\right]}\right\}
$$


where

$$
\mathrm{E}\left[R_{i j}\right]=\frac{N+1}{n_{i}+1} j
$$

and

$$
\operatorname{Var}\left[R_{i j}\right]=\frac{j}{n_{i}+1}\left(1-\frac{j}{n_{i}+1}\right) \frac{\left(N-n_{i}\right)(N+1)}{n_{i}+2} .
$$

Now, we investigate the behavior of the $k$-sample modified Baumgartner statistic $B_{k}^{*}$. We compare the power of the $B_{k}^{*}$ statistic with the Kruskal-Wallis test $W_{k}$, the $k$-sample Kolmogorov-Smirnov test $K S_{k}$, the $k$-sample Cramér-von Mises test $C V_{k}$, the $k$-sample Anderson-Darling test $A D_{k}$ and the $k$-sample Baumgartner statistic $B_{k}$. The $B_{k}$ statistic is defined by

$$
B_{k}=\frac{1}{k} \sum_{i=1}^{k}\left\{\frac{1}{n_{i}} \sum_{j=1}^{n_{i}} \frac{\left(R_{i j}-\alpha_{i j}\right)^{2}}{\beta_{i j}}\right\}
$$

where

$$
\alpha_{i j}=\frac{N}{n_{i}} j
$$

and

$$
\beta_{i j}=\frac{j}{n_{i}+1}\left(1-\frac{j}{n_{i}+1}\right) \frac{\left(N-n_{i}\right) N}{n_{i}} .
$$

Suppose that $F_{i}(x)$ is distributed as $N\left(\mu_{i}, \sigma_{i}^{2}\right), D E\left(\mu_{i}, \sigma_{i}\right), L\left(\mu_{i}, \sigma_{i}\right)$ and $\eta\left(\lambda_{i}\right)$, respectively. We examine the powers when the location and scale parameters are different, and the selected critical values are listed in Table 6 as estimated by simulation study.

Table 6: Critical values

\begin{tabular}{ccc|cc|cc|cc|cc}
\hline$n_{1}$ & $n_{2}$ & $n_{3}$ & $\operatorname{Pr}\left(B_{k} \geq b_{k}\right)$ & $b_{k}$ & $\operatorname{Pr}\left(B_{k} \geq b_{k}\right)$ & $b_{k}$ & $\operatorname{Pr}\left(B_{k}^{*} \geq b_{k}^{*}\right)$ & $b_{k}^{*}$ & $\operatorname{Pr}\left(B_{k}^{*} \geq b_{k}^{*}\right)$ & $b_{k}^{*}$ \\
\hline 10 & 7 & 5 & 0.010 & 2.989 & 0.050 & 2.120 & 0.010 & 3.170 & 0.050 & 2.213 \\
10 & 10 & 10 & 0.010 & 3.047 & 0.050 & 2.138 & 0.010 & 3.171 & 0.050 & 2.198 \\
\hline
\end{tabular}

The critical values were estimated by simulation study.

Table 7 to Table 10 are the results of the normal distribution, the double exponential distribution, the logistic distribution and the exponential distribution when $n_{1}=n_{2}=n_{3}$ or $n_{1} \neq n_{2} \neq n_{3}$. The significant level of this simulation is $5 \%$.

From Table 7 to Table 10, we can see that the power of the $B_{k}^{*}$ statistic is almost the same as the $B_{k}$ statistic when sample sizes are equal. However, the $B_{k}^{*}$ statistic is more efficient than the $B_{k}$ statistic for different sample sizes. For the location parameter, the $B_{k}^{*}$ statistic is almost the same as the $W_{k}$ statistic. For the scale parameter, the power of the $B_{k}^{*}$ statistic is higher than those of the $C V_{k}$ statistic and $A D_{k}$ statistic. Also, the power of the $B_{k}^{*}$ statistic is more suitable than that of the $K S_{k}$ statistic for location and scale parameters. 
A $K$-sample Baumgartner Statistic

Table 7-(a): The case of $n_{1}=n_{2}=n_{3}=10$ for $N\left(\mu_{i}, 1\right)$

\begin{tabular}{|c|c|c|c|c|c|}
\hline & & $\mu_{1}$ & $\mu_{2}$ & $\mu_{3}$ & \\
\hline & $0,0,0$ & $0,0,0.5$ & $0,0,1$ & $0,0.5,1$ & $0,1,2$ \\
\hline$W_{k}$ & 0.05 & 0.17 & 0.55 & 0.43 & 0.96 \\
\hline$K S_{k}$ & 0.05 & 0.14 & 0.45 & 0.34 & 0.91 \\
\hline$C V_{k}$ & 0.05 & 0.16 & 0.53 & 0.42 & 0.96 \\
\hline$A D_{k}$ & 0.05 & 0.17 & 0.54 & 0.42 & 0.96 \\
\hline$B_{k}$ & 0.05 & 0.16 & 0.53 & 0.41 & 0.95 \\
\hline$B_{k}^{*}$ & 0.05 & 0.16 & 0.53 & 0.41 & 0.95 \\
\hline
\end{tabular}

Table 7-(b): The case of $n_{1}=n_{2}=n_{3}=10$ for $N\left(0, \sigma_{i}^{2}\right)$

\begin{tabular}{|c|c|c|c|c|c|}
\hline & \multicolumn{2}{|r|}{$\sigma_{1}$} & $\sigma_{2}$ & \multicolumn{2}{|l|}{$\sigma_{3}$} \\
\hline & $1,1,1$ & $1,5,5$ & $1,5,10$ & $1,10,10$ & $1,10,20$ \\
\hline$W_{k}$ & 0.05 & 0.07 & 0.08 & 0.08 & 0.08 \\
\hline$K S_{k}$ & 0.05 & 0.23 & 0.32 & 0.39 & 0.46 \\
\hline$C V_{k}$ & 0.05 & 0.22 & 0.34 & 0.35 & 0.47 \\
\hline$A D_{k}$ & 0.05 & 0.25 & 0.45 & 0.39 & 0.60 \\
\hline$B_{k}$ & 0.05 & 0.32 & 0.52 & 0.59 & 0.76 \\
\hline$B_{k}^{*}$ & 0.05 & 0.32 & 0.50 & 0.58 & 0.75 \\
\hline
\end{tabular}

Table 7-(c): The case of $n_{1}=10, n_{2}=7, n_{3}=5$ for $N\left(\mu_{i}, 1\right)$

\begin{tabular}{cccccc}
\hline & \multicolumn{1}{c}{$\mu_{1}$} & $\mu_{2}$ & $\mu_{3}$ & \\
\cline { 2 - 6 } & $0,0,0$ & $0,0,0.5$ & $0,0,1$ & $0,0.5,1$ & $0,0.5,1$ \\
\hline$W_{k}$ & $\mathbf{0 . 0 5}$ & 0.11 & 0.32 & 0.30 & 0.85 \\
$K S_{k}$ & $\mathbf{O . O 5}$ & 0.10 & 0.28 & 0.26 & 0.76 \\
$C V_{k}$ & $\boldsymbol{O . O 5}$ & 0.11 & 0.31 & 0.29 & 0.83 \\
$A D_{k}$ & $\boldsymbol{O . O 5}$ & 0.11 & 0.32 & 0.29 & 0.84 \\
$B_{k}$ & $\boldsymbol{O . O 5}$ & 0.08 & 0.22 & 0.23 & 0.78 \\
$B_{k}^{*}$ & $\boldsymbol{O . O 5}$ & 0.10 & 0.28 & 0.29 & 0.83 \\
\hline
\end{tabular}

Table 7-(d): The case of $n_{1}=10, n_{2}=7, n_{3}=5$ for $N\left(0, \sigma_{i}^{2}\right)$

\begin{tabular}{|c|c|c|c|c|c|}
\hline & & $\sigma_{1}$ & $\sigma_{2}$ & $\sigma_{3}$ & \\
\hline & $1,1,1$ & $1,5,5$ & $1,5,10$ & $1,10,10$ & $1,10,20$ \\
\hline$W_{k}$ & 0.05 & 0.11 & 0.12 & 0.12 & 0.12 \\
\hline$K S_{k}$ & 0.05 & 0.23 & 0.30 & 0.35 & 0.40 \\
\hline$C V_{k}$ & 0.05 & 0.22 & 0.29 & 0.32 & 0.38 \\
\hline$A D_{k}$ & 0.05 & 0.28 & 0.40 & 0.40 & 0.51 \\
\hline$B_{k}$ & 0.05 & 0.31 & 0.40 & 0.49 & 0.56 \\
\hline$B_{k}^{*}$ & 0.05 & 0.33 & 0.43 & 0.51 & 0.60 \\
\hline
\end{tabular}


MURAKAMI

Table 8-(a): The case of $n_{1}=n_{2}=n_{3}=10$ for $D E\left(\mu_{i}, 1\right)$

\begin{tabular}{|c|c|c|c|c|c|}
\hline & & $\mu_{1}$ & $\mu_{2}$ & $\mu_{3}$ & \\
\hline & $0,0,0$ & $0,0,0.5$ & $0,0,1$ & $0,0.5,1$ & $0,1,2$ \\
\hline$W_{k}$ & 0.05 & 0.14 & 0.42 & 0.33 & 0.84 \\
\hline$K S_{k}$ & 0.05 & 0.14 & 0.41 & 0.32 & 0.81 \\
\hline$C V_{k}$ & 0.05 & 0.15 & 0.43 & 0.35 & 0.86 \\
\hline$A D_{k}$ & 0.05 & 0.14 & 0.42 & 0.34 & 0.84 \\
\hline$B_{k}$ & 0.05 & 0.14 & 0.42 & 0.33 & 0.84 \\
\hline$B_{k}^{*}$ & 0.05 & 0.14 & 0.42 & 0.34 & 0.84 \\
\hline
\end{tabular}

Table 8-(b): The case of $n_{1}=n_{2}=n_{3}=10$ for $D E\left(0, \sigma_{i}\right)$

\begin{tabular}{cccccc}
\hline & \multicolumn{1}{c}{$\sigma_{1}$} & $\sigma_{2}$ & $\sigma_{3}$ & \\
\cline { 2 - 6 } & $1,1,1$ & $1,5,5$ & $1,5,10$ & $1,10,10$ & $1,10,20$ \\
\hline$W_{k}$ & $\mathbf{0 . 0 5}$ & 0.07 & 0.07 & 0.07 & 0.08 \\
$K S_{k}$ & $\mathbf{O . 0 5}$ & 0.16 & 0.25 & 0.30 & 0.38 \\
$C V_{k}$ & $\mathbf{O . 0 5}$ & 0.17 & 0.25 & 0.28 & 0.37 \\
$A D_{k}$ & $\mathbf{O . 0 5}$ & 0.19 & 0.31 & 0.31 & 0.46 \\
$B_{k}$ & $\mathbf{O . 0 5}$ & 0.21 & 0.34 & 0.43 & 0.59 \\
$B_{k}^{*}$ & $\mathbf{0 . 0 5}$ & 0.21 & 0.34 & 0.43 & 0.58 \\
\hline
\end{tabular}

Table 8-(c): The case of $n_{1}=10, n_{2}=7, n_{3}=5$ for $D E\left(\mu_{i}, 1\right)$

\begin{tabular}{cccccc}
\hline & & $\mu_{1}$ & $\mu_{2}$ & $\mu_{3}$ & \\
\cline { 2 - 6 } & $0,0,0$ & $0,0,0.5$ & $0,0,1$ & $0,0.5,1$ & $0,1,2$ \\
\hline$W_{k}$ & $\boldsymbol{O . 0 5}$ & 0.10 & 0.24 & 0.23 & 0.65 \\
$K S_{k}$ & $\mathbf{O . 0 5}$ & 0.10 & 0.24 & 0.24 & 0.65 \\
$C V_{k}$ & $\boldsymbol{O . 0 5}$ & 0.10 & 0.25 & 0.24 & 0.68 \\
$A D_{k}$ & $\boldsymbol{O . 0 5}$ & 0.10 & 0.25 & 0.24 & 0.68 \\
$B_{k}$ & $\mathbf{O . 0 5}$ & 0.07 & 0.18 & 0.19 & 0.61 \\
$B_{k}^{*}$ & $\boldsymbol{O . 0 5}$ & 0.10 & 0.24 & 0.24 & 0.68 \\
\hline
\end{tabular}

Table 8-(d): The case of $n_{1}=10, n_{2}=7, n_{3}=5$ for $D E\left(0, \sigma_{i}\right)$

\begin{tabular}{|c|c|c|c|c|c|}
\hline & & $\sigma_{1}$ & $\sigma_{2}$ & $\sigma_{3}$ & \\
\hline & $1,1,1$ & $1,5,5$ & $1,5,10$ & $1,10,10$ & $1,10,20$ \\
\hline$\overline{W_{k}}$ & 0.05 & 0.10 & 0.11 & 0.11 & 0.12 \\
\hline$K S_{k}$ & 0.05 & 0.18 & 0.24 & 0.28 & 0.34 \\
\hline$C V_{k}$ & 0.05 & 0.18 & 0.23 & 0.27 & 0.32 \\
\hline$A D_{k}$ & 0.05 & 0.22 & 0.31 & 0.34 & 0.43 \\
\hline$B_{k}$ & 0.05 & 0.23 & 0.29 & 0.39 & 0.46 \\
\hline$B_{k}^{*}$ & 0.05 & 0.24 & 0.32 & 0.41 & 0.49 \\
\hline
\end{tabular}


A $K$-sample Baumgartner Statistic

Table 9-(a): The case of $n_{1}=n_{2}=n_{3}=10$ for $L\left(\mu_{i}, 1\right)$

\begin{tabular}{|c|c|c|c|c|c|}
\hline & & $\mu_{1}$ & $\mu_{2}$ & \multicolumn{2}{|l|}{$\mu_{3}$} \\
\hline & $0,0,0$ & $0,0,0.5$ & $0,0,1$ & $0,0.5,1$ & $0,1,2$ \\
\hline$W_{k}$ & 0.05 & 0.09 & 0.22 & 0.18 & 0.57 \\
\hline$K S_{k}$ & 0.05 & 0.08 & 0.19 & 0.15 & 0.48 \\
\hline$C V_{k}$ & 0.05 & 0.09 & 0.22 & 0.17 & 0.56 \\
\hline$A D_{k}$ & 0.05 & 0.09 & 0.22 & 0.17 & 0.55 \\
\hline$B_{k}$ & 0.05 & 0.09 & 0.22 & 0.17 & 0.55 \\
\hline$B_{k}^{*}$ & 0.05 & 0.09 & 0.22 & 0.17 & 0.55 \\
\hline
\end{tabular}

Table 9-(b): The case of $n_{1}=n_{2}=n_{3}=10$ for $L\left(0, \sigma_{i}\right)$

\begin{tabular}{cccccc}
\hline & & $\sigma_{1}$ & $\sigma_{2}$ & $\sigma_{3}$ & \\
\cline { 2 - 6 } & $1,1,1$ & $1,5,5$ & $1,5,10$ & $1,10,10$ & $1,10,20$ \\
\hline$W_{k}$ & $\mathbf{0 . 0 5}$ & 0.07 & 0.08 & 0.08 & 0.08 \\
$K S_{k}$ & $\mathbf{0 . 0 5}$ & 0.21 & 0.31 & 0.37 & 0.45 \\
$C V_{k}$ & $\mathbf{0 . 0 5}$ & 0.21 & 0.31 & 0.33 & 0.45 \\
$A D_{k}$ & $\mathbf{0 . 0 5}$ & 0.24 & 0.41 & 0.38 & 0.56 \\
$B_{k}$ & $\mathbf{0 . 0 5}$ & 0.29 & 0.47 & 0.55 & 0.72 \\
$B_{k}^{*}$ & $\mathbf{0 . 0 5}$ & 0.29 & 0.46 & 0.54 & 0.71 \\
\hline
\end{tabular}

Table 9-(c): The case of $n_{1}=10, n_{2}=7, n_{3}=5$ for $L\left(\mu_{i}, 1\right)$

\begin{tabular}{cccccc}
\hline & & $\mu_{1}$ & $\mu_{2}$ & $\mu_{3}$ & \\
\cline { 2 - 6 } & $0,0,0$ & $0,0,0.5$ & $0,0,1$ & $0,0.5,1$ & $0,1,2$ \\
\hline$W_{k}$ & $\mathbf{0 . 0 5}$ & 0.07 & 0.13 & 0.13 & 0.39 \\
$K S_{k}$ & $\mathbf{0 . 0 5}$ & 0.07 & 0.13 & 0.12 & 0.36 \\
$C V_{k}$ & $\mathbf{O . 0 5}$ & 0.07 & 0.14 & 0.13 & 0.39 \\
$A D_{k}$ & $\mathbf{0 . 0 5}$ & 0.07 & 0.14 & 0.13 & 0.39 \\
$B_{k}$ & $\mathbf{O . 0 5}$ & 0.06 & 0.10 & 0.10 & 0.32 \\
$B_{k}^{*}$ & $\mathbf{0 . 0 5}$ & 0.07 & 0.13 & 0.13 & 0.39 \\
\hline
\end{tabular}

Table 9-(d): The case of $n_{1}=10, n_{2}=7, n_{3}=5$ for $L\left(0, \sigma_{i}\right)$

\begin{tabular}{cccccc}
\hline & & $\sigma_{1}$ & $\sigma_{2}$ & $\sigma_{3}$ & \\
\cline { 2 - 6 } & $1,1,1$ & $1,5,5$ & $1,5,10$ & $1,10,10$ & $1,10,20$ \\
\hline$W_{k}$ & $\boldsymbol{O . 0 5}$ & 0.11 & 0.11 & 0.12 & 0.12 \\
$K S_{k}$ & $\mathbf{O . 0 5}$ & 0.22 & 0.29 & 0.33 & 0.39 \\
$C V_{k}$ & $\mathbf{O . 0 5}$ & 0.21 & 0.28 & 0.30 & 0.36 \\
$A D_{k}$ & $\boldsymbol{O . 0 5}$ & 0.27 & 0.37 & 0.39 & 0.49 \\
$B_{k}$ & $\boldsymbol{O . 0 5}$ & 0.29 & 0.37 & 0.46 & 0.54 \\
$B_{k}^{*}$ & $\boldsymbol{O . 0 5}$ & 0.30 & 0.40 & 0.49 & 0.58 \\
\hline
\end{tabular}


Table 10-(a): The case of $n_{1}=n_{2}=n_{3}=10$ for $\eta\left(\lambda_{i}\right)$

\begin{tabular}{cccccc}
\hline & & $\lambda_{1}$ & $\lambda_{2}$ & $\lambda_{3}$ & \\
\cline { 2 - 6 } & $1,1,1$ & $1,2,2$ & $1,2,3$ & $1,3,3$ & $1,3,5$ \\
\hline$W_{k}$ & $\mathbf{0 . 0 5}$ & 0.23 & 0.42 & 0.52 & 0.75 \\
$K S_{k}$ & $\mathbf{O . 0 5}$ & 0.18 & 0.34 & 0.43 & 0.67 \\
$C V_{k}$ & $\mathbf{O . 0 5}$ & 0.23 & 0.40 & 0.51 & 0.74 \\
$A D_{k}$ & $\mathbf{O . 0 5}$ & 0.23 & 0.41 & 0.51 & 0.75 \\
$B_{k}$ & $\mathbf{0 . 0 5}$ & 0.24 & 0.42 & 0.54 & 0.76 \\
$B_{k}^{*}$ & $\mathbf{0 . 0 5}$ & 0.23 & 0.41 & 0.53 & 0.77 \\
\hline
\end{tabular}

Table 10-(b): The case of $n_{1}=10, n_{2}=7, n_{3}=5$ for $\eta\left(\lambda_{i}\right)$

\begin{tabular}{|c|c|c|c|c|c|}
\hline & & $\lambda_{1}$ & $\lambda_{2}$ & $\lambda_{3}$ & \\
\hline & $1,1,1$ & $1,2,2$ & $1,2,3$ & $1,3,3$ & $1,3,5$ \\
\hline$W_{k}$ & 0.05 & 0.20 & 0.31 & 0.43 & 0.60 \\
\hline$K S_{k}$ & 0.05 & 0.18 & 0.28 & 0.38 & 0.55 \\
\hline$C V_{k}$ & 0.05 & 0.20 & 0.31 & 0.43 & 0.59 \\
\hline$A D_{k}$ & 0.05 & 0.20 & 0.32 & 0.43 & 0.61 \\
\hline$B_{k}$ & 0.05 & 0.19 & 0.28 & 0.43 & 0.58 \\
\hline$B_{k}^{*}$ & 0.05 & 0.22 & 0.33 & 0.47 & 0.63 \\
\hline
\end{tabular}

\section{Conclusion and discussion}

In this paper, we propose a modified Baumgartner statistic $B^{*}$ and a $k$-sample Baumgartner statistic $B_{k}^{*}$. By simulation studies, we find that the $B^{*}$ statistic is superior to the $B$ statistic. Then, the $B^{*}$ statistic is generalized from a two-sample to a $k$-sample statistic. By a power comparison, the $B_{k}^{*}$ statistic is more efficient than the $W_{k}$ statistic, the $K S_{k}$ statistic, the $C V_{k}$ statistic, the $A D_{k}$ statistic and the $k$-sample Baumgartner statistic $B_{k}$. Therefore, we can say that our new statistic is more competent than the $W_{k}$ statistic, the $K S_{k}$ statistic, the $C V_{k}$ statistic, the $A D_{k}$ statistic and the $B_{k}$ statistic. In future work, one of the problems is deriving the asymptotic distribution of $k$-sample Baumgartner statistic.

\section{Acknowledgements}

The author would like to thank the editor and the referees for valuable comments and suggestions.

\section{REFERENCES}

Anderson, T. W. (1962). On the distribution of the two-sample Cramér-von Mises criterion. Annals of Mathematical Statistics 33, 1148-1159.

Arnold, B. C., Balakrishnan, N. and Nagaraja, H. N. (1992). A First Course in Order Statistics. New York: John Wiley \& Sons.

Baumgartner, W., Weiß, P. and Schindler, H. (1998). A nonparametric test for the general two-sample problem. Biometrics 54, 1129-1135.

Hájek, J., Šidák, Z. and Sen, P. K. (1999). Theory of Rank Tests, 2nd edition. San Diego: Academic Press. 
Hollander, M. and Wolfe, D. A. (1999). Nonparametric Statistical Methods, 2nd edition. New York: John Wiley \& Sons.

Kiefer, J. (1959). $K$-sample analogues of the Kolmogorov-Smirnov and Cramér-von Mises tests. Annals of Mathematical Statistics 30, 420-447.

Neuhäuser, M. (2000). An exact two-sample test based on the Baumgartner-Weiss-Schindler statistic and a modification of Lepage's test. Communications in Statistics - Simulation and Computation 29, 67-78.

Neuhäuser, M. (2001). One-side two-sample and trend tests based on a modified Baumgartner-Weiss-Schindler statistic. Journal of Nonparametric Statistics 13, 729-739.

Neuhäuser, M. (2003). A note on the exact test based on the Baumgartner-Weiß-Schindlelr statistic in a presence of ties. Computational Statistics and Data Analysis 42, 561-568.

Pettitt, A. N. (1976). A two-sample Anderson-Darling rank statistic. Biometrka 63, 161-168.

Scholz, W. and Stephens, M. (1987). K-sample Anderson-Darling tests. Journal of the American Statistical Association 82, 918-924.

(Received February 2005, Accepted December 2005) 